\title{
CONSTRUCTION OF \\ CORPUS-BASED SYNTACTIC RULES FOR ACCURATE SPEECH RECOGNITION
}

\author{
JUNKO HOSAKA TOSHIYUKI TAKEZAWA \\ ATR Interpreting Telephony Research Laboratories \\ Hikaridai 2-2, Seika-cho, Soraku-gun \\ Kyoto 619-02, Japan \\ hosaka@atr-la.atr.co.jp \\ takezawa@atr-la.atr.co.jp
}

\begin{abstract}
This paper describes the syntactic rules which are applied in the Japanese speech recognition module of a speech-to-speech translation system. Japanese is considered to be a free word/phrase order language. Since syntactic rules are applied as constraints to reduce the search space in speech recognition, applying rules which take into account all possible phrase orders can have almost the same effect as using no constraints. Instead, we take into consideration the recognition weaknesses of certain syntactic categories and treat them precisely, so that a minimal number of rules can work most effectively. In this paper we first examine which syntactic categories are easily misrecognized. Second, we consult our dialogue corpus, in order to provide the rules with great generality. Based on both studies, we refine the rules. Fin nally, we verify the validity of the refinement through speech recognition experiments.
\end{abstract}

\section{Introduction}

We are developing the Spoken Language TRANSlation system (SL-TRANS)[1], in which both speech recognition processing and natural language processing are integrated. Currently we are studying automatic speech translation from Japanese into English in the domain of dialogues with the reception service of an international conference office. In this framework we are constructing syntactic rules for recognition of $J$ apanese speech.

In speech recognition, the most significant concern is raising the recognition accuracy. For that purpose, applying linguistic information turns out to be promising. Various approaches have been taken, such as using stochastic models[2], syntactic rules[3], semantic information[4] and discourse plans[5]. Among stochastic models, the bigram and trigram succeeded in achieving a high recognition accuracy in languages that have a strong tendency toward a standard word order, such as English. On the contrary, Japanese belongs to free word order languages[6]. For such a language, semantic information is more adequate as a constraint. However, building semantic constraints for a large vocabulary needs a tremendous amount of data. Currently, our data consist of dialogues between the conference registration office and prospective conference participants with approximately 199,000 words in telephone conversations and approximately 72,000 words in keyboard conversations. But our data are still not sufficient to build appropriate semantic constraints for sentences with 700 distinct words. Processing a discourse plan requires excessive calculation and the study of discourse itself must be further developed to be applicable to speech recognition. On the other hand, syntax has been studied in more detail and makes increasing the vocabulary easier.

As we are working on spoken language, we try to reflect real language usage. For this purpose, a stochastic approach beyond trigrams, namely stochastic sentence parsing[7], seems most promising. Ideally, syntactic rules should be generated automatically from a large dialogue corpus and probabilities should also be automatically assigned to each node. But to do so, we need underlying rules. Moreover, coping with phoneme perplexity, which is crucial to speech recognition, with rules created from a dialogue corpus, requires additional research [8].

In this paper we propose taking into account the weaknesses of the speech recogniton system in the earliest stage, namely when we construct underlying syntactic rules. First, we examined the speech recognition results to determine which syntactic categories tend to be recognized erroneously. Second, we utilized our dialogue corpus [9] to support the refinement of rules concerning those categories. $A$ s examples, we discuss formal nouns ${ }^{1}$ and conjunctive postpositions ${ }^{2}$. Finally, we carried out a speech recognition experiment with the refined rules to verify the validity of our approach.

\footnotetext{
${ }^{1}$ Formal nouns: keistuki-meishi in Japanese.

${ }^{2}$ Conjunctive postpositions : setsuzoku-joshi in Japanese.
} 


\section{Issues in HMM-LR Speech Recognition}

In the Japanese speech recognition module of our experimental system the combination of generalized I,R parsing and Hidden Markov Model (HMM) is realized as $11 \mathrm{MM}-\mathrm{LR}$ [10]. The system predicts phonemes by using an L,R parsing table and drives HMM phoneme verifiers to detect/verify t,lem withont any intervening structure, such as a phoneme lattice.

The speech recognition unit is a Japanese bunsetsu, which roughly corresponds to a phrase and is the next largest unit after the word. 'The ending of the bunsetsu (phrase) is ustally marked by a breath point. This justifies its treatment as a distinct unit. A Japanese phrase consists of one independent word (e.g. noun, adverb, verb) and zero, one or more than one dependent words (e.g. postposition, auxiliary verb). The number of words in a pirase ranges fron 1 to 14 , and the mean number is about 3 , according to our dialogue corpus.

We will clarify the wcaknesses of $11 M M-L R$ speech recognition both in phrases and in sentences.

\subsection{Phrase Recognition Errors}

We examined which syntactic categories tend to be erroneously recognized, when using II M M LR phrase speech recognition. For this purpose, we applied syntactic rules containing no constraints on word sequences ${ }^{3}$. This means that any word can follow any word.

Examples (1) and (2) show the results of IIM M-J, R Japanese speech recognition ${ }^{4}$. The uttered phoneme strings are enclosed in $\mid$ l.

\section{(1) $\mid$ sochirawa $\mid$ (this, that)}

1: sochira-wa
2: sochira-wa-hu
3: sochira-hu-wa
4: sochixa-hu-wa-hu
5: sochixa-wa-hu-hu

(2) |aringatougozaimasu| (thank you)

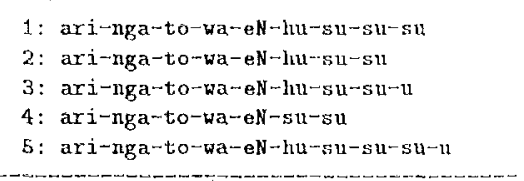

\footnotetext{
3 apanese verbs, adjectives, etc, are always inllected when used. In syntactic rules containing no word seguence constraints, inflected verbs, inflected actjectives, etc. are consiclered to be words.

The maximal amouni of whole bean width, t]e global beam width, is set for 16 and the Inaximal bearn width of each branch, the local bean width, 10 .
}

In the examples, the symbols $>,-$, ng and $\mathrm{N}$ have special meaning:

- A correctly recognized phrise is marked with $>$.

- $\Lambda$ word boundary is marked with -

- A nusalized /g/ is transcribed ng.

- A syllabic nasal is transcribed $\mathrm{N}$.

In (1), after recognizing the first word, the systcm selected subsequent words solely to produce a phoneme string similar to the original utterance.

(2) is an exarnple of plurase recognition which failed. In this example tou was erroneously recognized as to. Sulisequently, no further correct words were selected.

Txamples (1) and (2) both show that IIMM-l, R tends to select words consisting of extremely few phonernes when it fails in word recognition. To avoid this problem, precise rules should be written for sequences of words with small numbers of phonenes. In Japancse, postpositions(e.g. ga, o, ni), wh-pronouns(e.g. itsu, nani, dare)[11], numerals(e.g. ichi, ni, san) and certain nouns(e.g. kata, mono) particularly fit this description.

\subsection{Sentence Recognition Errors}

To exanme the error tendency of sentence speech recognition we applied a two-step method[12]. First, we applied phrase rules to the HMM-LR speech recognition ${ }^{5}$. Second, we applied phrase-based sentence rules to the phrase candidates as at post-filter, in order to obtain sentence candidates, while filtering out unacceptable candidates. We experimented with the 35.3 phrases making up 137 sentences. The recognition rate for the top candidates was $68.3 \%$ by exact string matching, and for the top 5 candidates $95.5 \%$

Based on the top 5 phrase candidates, we conducted a sentence experinent. In this experinent we applied loosely constrained sentence rules. Witl these rules, approximately $80 \%$ of all the possible combinations of phrase candidates were accejted. Following are cxamples which did not exactly match the uttered sentences ${ }^{6}$. Notice that misrecognized words consist of a relatively stmall number of phonemes, an we have seen in scction 2.1 .

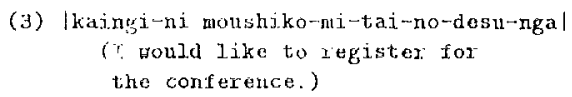

3a: kaingi-ni moushiko-mi-tai-N-desu-nga

Ah: kaingi-ni mousliko-mi-tai-no-desu-ka

(4) |kochira-wa kaingizinukyoku-desu|

\footnotetext{
SThe glokal bean width is bet for 100 and the local beam width 10 .

6 Since the plirese candidntes are obtained by the HMM-IJT speech reconguition, word boundaries are sheady marked by -
} 
(This is the conference office.)

4a: kata-wa kaingizimukyoku-desu

(5) |doumo aringat-ou-gozaima-shj-tal (Thank you very much.)

5a: go-o aringat-ou-gozaima-shi-ta

5b: go-mo axingat-ou-gozaima-shi-ta

5c: mono aringat-ou-gozaima-shi-ta

(6) |gozyuusho-to onamae-o onengai-shi-masu| (Can I have your name and address?)

6a: gozyulsho-to onamae-o onengai-shi-masu-shi

Though the phoneme string in $3 a$ is different from the uttered phoneme string, the difference between no and $\mathbf{N}$ in meaning is minor, and has no effect on translation with the current technique. While (3) is affirmative, $3 \mathrm{~b}$ is interrogative, which is indicated by the sentence final postposition ka. This cannot be treated with sentence rules. To handle this problem, we need dialogue management.

The uttered phrase kochira-wa in (4), meaning "this," was recognized erroneously as kata-wa in 4a, meaning "person." The word kata belongs to the formal noun group, a kind of noun which should be modified by a verbal phrase [13]. Sentence $4 a$ is acceptable, if modified by a verbal phrase, as in $4 a^{\prime}$ :

4a': midori-no seihuku-o kiteirn kata-wa kaigizimukyoku-desu

(The person who is wearing a green uniform is [with] the conference office.)

This is also true of the phrase mono in $5 \mathrm{c}$ meaning "thing," which was erroneously recognized instead of doumo meaning "very much":

5c': kouka-na mono aringat-on-gozaima-shi-ta

(Thank you for the expensive thing.)

In sentence candidates $5 \mathrm{a}$ and $5 \mathrm{~b}$, the numeral go, meaning "five," is used. These sentences may seem strange at first glance, but in a situation such as playing cards, these sentences are quite natural. If someone plays a 5 when you need one, you would say: "Thanks for the five." Similarly, when you need a 3 and $\mathrm{a}$, and someone plays a 3 and after that someone else plays a 5, you would say: "Thanks for the five, too."

In the sentence candidate $6 \mathrm{a}$, the conjunctivepostposition (conj-pp) shi is used sentence finally. In principle, a conj-pp combines two sentences, functioning like a conjunction, such as "while" and "though," and is used in the middle of a sentence.
Frroneous sentence recognition such as in the case of $3 \mathrm{a}-\mathrm{b}$ cannot be treated by sentence rules. Therefore, we are trying to cope with erroneous recognition, as seen in sentence candidates $4 \mathrm{a}, 5 \mathrm{a}-\mathrm{c}$ and $6 \mathrm{a}$, with sentence rules.

\section{Dealing with Speech Recog- nition Errors}

We are going to deal with sentences containing the following phrases:

- Phrases with formal nouns

- Phrases with nunierals

- Phrases with conj-pps used in the sentence final position

In order to decide how to cope with the above problems, we used our dialogue corpus. Currently we have 177 keyboard conversations consisting of approximately 72,000 words and 181 telephone conversations consisting of approximately 199,000 words $^{7}$. We regard keyboard conversations as representing written Japanese and telephone conversations as representing spoken Japanese. When retrieving the dialogue corpus, we always compare written and spoken Japanese, in order to clarify the features of the latter. We examined the actual usage of formal nouns as well as that of conj-pps.

\subsection{Formal Nouns}

We examined the behavior of formal nouns, such as koto and mono. Formal nouns are considered to be a kind of noun which lacks the content usually found in common nouns such as "sky" or "apple." They function similarly to relative pronouns and therefore are used with a verbal modifier[13], as in examples 7 and 8 :

7 : kinou itta koto-wa torikeshitai.

(I would like to take back what I said yesterday.)

8: nedan-ga takai mono-ga shitsu-ga ii wakedewanai.

(It is not always true that an expensive thing has good quality.)

In examples 7 and 8 , the formal nouns, koto and mono, are modified by kinou itta (yesterday said) and nedan-ga takai (price expensive), respectively. But it is also true that these nouns behave like common nouns and can be used without any verbal nodifier, as in examples 9 and 10 :

9 : sore-wa koto desu ne.

${ }^{7}$ The dialogue corpus is growing constantly. When we retrieved formal nouns, we had 113 keyboard conversations and 96 teiephone conversations. 
(lt is a grave matter.)

\section{0: mono-wa tashika-da.}

('This stuff is trustworthy.)

Considering the examples 7-10, we could define two kinds of usage for formal nouns. 'This distinction is applicable to sentence analysis, but is meaningless from the standpoint of applying syutactic rules as constraints.

\subsubsection{Formal Nouns in the Corpus}

In our dialogue corpus, koto, mono, hou and kata are the most frequently used formal nouns. Table 1 shows how often the formal nouns are used with a verbal modifier. We have also retrieved formal nouns used in the sentence initial position, as in example 10.

Table 1: Formal Nouns

\begin{tabular}{|l|r|r|r|r|}
\hline & \multicolumn{2}{|c|}{ Keyboard } & \multicolumn{2}{|c|}{ Telephone } \\
\cline { 2 - 5 } & Freq. & $\%$ & Freq. & $\%$ \\
\hline With Verb. Mod. & 358 & 63 & 774 & 40 \\
Without Verb. Mod. & 214 & 37 & 1,145 & 60 \\
Sent. Initial & 0 & 0 & 0 & 0 \\
\hline Total & 572 & 100 & 1,919 & 100 \\
\hline
\end{tabular}

Table 1 indicates that the coverage reaches $63 \%$ in written Japanese, when we allow only formal nouns preceded by a verbal modifier in the syntactic rules. Iowever, the coverage remains at $40 \%$, which is less than half, in the spoken Japanese we are dealing with. Wo have further examined those sentences in which formal nouns are not modified by verbals. Most of them are modified by phrases consisting of a noun and postposition no, which approximately corresponds to "of." Further, some are modified by plirases consisting of a verb followed by postpositions to and no. Others are modified by words which can be used exclusively as nominal modifiers such as donna (what kind of) and sono (that). We found only one example in the keyboard conversation in which a formal noun is not modified at all:

11 : osoraku kyouju-ni koto-no shidai-o tsutacru koto-ga ii-to omoimasu.

(It might be good if you tell the professor how the thing is going.)

In our dialogue corpus we found 2,491 phrases containing the fornal nouns koto, mono, hou and kata. Out of 2,491 examples, there is only one which is not modified at all. If we define formal nouns as those which are always moditied in some manner, i.e. even if we do not allow formal nouns to be used alone, the coverage still exceeds $99 \%$. Since the occurrence rate of formal nouns without any modifier is very low, we can treat the usage of formal nouns (as in examples 9-11) as semi-frozen expressions.

\subsection{Conjunctive Postpositions}

Japanese postpositions such as $g a$, o and $n$, which function as case markers, are usually attached to nominals. Different from this kind of pastposition, conj-pps such as ga, te and ba are used after verbals. Conj-pps combine two clauses, functioning similarly to conjunctions such as "because" and "while", and are thus often used in the tididdle of a sentence, as in example 12. Jut they can also be used in the sentence final position, as in eximple 13 .

12:

kaigi-ni motsikomi-tai-no-desu-ga, DAT

mousikomiyousi-o ookurikudasai.

AKK

Mecause I would like to apply for the conference, please send me a registration form.

13:

kajgi-ni mousikomi-tai-no-desu-ga. DAT

I woukd like to apply for the conference, ...

Hxample 13 sounds vague, if uttered in isolation. There should follow some additional words to express the complete meaning. Sentences finishing with a conj-pp leave the interpretation to the hearer. And, in gencral, the hearer can correctly interpret the sentence from the context. Understanding conj-pps, therefore, plays an important role in treating spoken Japanese.

\subsubsection{Sentence Final Conj-pps in the Corpus}

In the dialogue corpus the following conj-pjs are used:

ga (becanse, while), node and nde (becausc), te and de (and), kara (because, after), keredomo, keredo, kedo and kedomo (though, but), shi (and, and then), monode (because), tara (if), to (if, when), ba (if) and nagara (while).

Table 2 show: conj-pos used sentence finally.

According to 'lable 2, the conj-pp ga is the one most used in keyboard conversations. While the usage of conj-pps in keyboard conversations is heavily concentrated on ga with all occurrence rate of $85 \%$, it is more balanced in telephone conversations. In addition to ga $(38 \%)$, keredomo (30\%) and conj-pps which carry a similar meaning such as keredo, kedo and kedono are frequently used. In telephonc conversations, node (13\%) is also frequently used. 'Ireating only the six conj-pps in sentence final position, the coverage reaches $91 \%$ for spoken $J$ ajpanese. J)ifferentiating conj-pps which can be used in sentence final position from those which can be used only in the middle of a sentence is also supported by the speech recognition results[14]. 'The conj-pps shi and cha are especially subject to erroneous recognition. 
Table 2: Sentence Final Conj-pps

\begin{tabular}{|l|r|r|r|r|}
\hline \multirow{2}{*}{ Conj-pp } & \multicolumn{2}{|c|}{ Keyboard } & \multicolumn{3}{|c|}{ Telephone } \\
\cline { 2 - 5 } & Irequency & $\%$ & Frequency & $\%$ \\
\hline ga & 197 & 85 & 274 & 38 \\
\hline node & 11 & 5 & 96 & 13 \\
\hline$n d e$ & 0 & 0 & 5 & 1 \\
\hline te & 8 & 3 & 23 & 3 \\
\hline de & 0 & 0 & 1 & 0 \\
\hline kara & 6 & 3 & 14 & 2 \\
\hline keredomo & 5 & 2 & 212 & 30 \\
\hline keredo & 1 & 0 & 18 & 3 \\
\hline kedo & 1 & 0 & 12 & 2 \\
\hline kedomo & 0 & 0 & 37 & 5 \\
\hline shi & 2 & 1 & 10 & 1 \\
\hline monode & 1 & 0 & 0 & 0 \\
\hline tara & 0 & 0 & 5 & 1 \\
\hline to & 0 & 0 & 2 & 0 \\
\hline ba & 0 & 0 & 2 & 0 \\
\hline unagara & 0 & 0 & 1 & 0 \\
\hline \hline
\end{tabular}

\subsection{Syntactic Rules for Speech Recog- nition}

Based on the corpus retrieval we decided to deal with formal nouns and conj-pos as described below. And we decided to treat numerals only in a restricted en. vironment, because they are significant noise factors in speech recognition ${ }^{8}$ :

- Phrases with formal nouns must be modified.

- Phrases witl mumerals can be used only in certain enviromments. Numerals are allowed in addresses, telephone numbers, dates and prices. Japanese numerals consist of an extremely small number of phonemes, e.g. ichi, $n i, \operatorname{san}(1,2,3)$ and are therefore especially easy to misrecognize? ${ }^{9}$. Thus, they should be strongly constrained. The domain we have chosen is limited to djalogues between an international conference receptionist and prospective participants and we are going to deal only with the anticipated usage in the domain. Another condition, such as playing cards, will be treated when speecl recognition is further improved.

- We classify conj-pps into two groups: conj-pps which can be used in the sentence final position as well as in the middle of a sentence, and conjpps which can be used only in the middle of a sentence.

We refined the loosely constrained syutactic rules introduced in section 2.2. In the new version of the sentence rules, formal nouns, numerals and conj-pps are more precisely treated. In the following, we explain the rules for formal nouns and conj-pps.

\footnotetext{
${ }^{6}$ See Figure 2.

INunbers greater than ten are in principle the combination of basic numbers.
}

The format for syntactic rules is as follows:

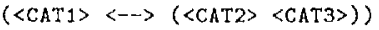

Nonterminals are surrounded by $<>^{10}$. The above rule indicates that CAT1 consists of CAT2 and CAT3. To make the distinction between phrase categories which are terminals in phrase-based sentence rules and those which are not, we will write the former all in lower-case.

In the process of sentence construction, phrases containing a formal noun np-formal are treated as follows ${ }^{11}$ :

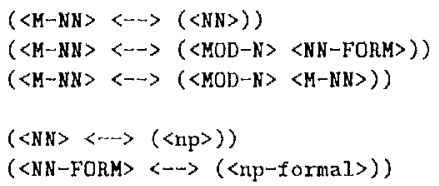

'The above rules say that noun phrases $H-N N$ can, in principle, be modified by some modifier MOD-N. In the case of a common noun $N N$, the phrase can be modified but need not be. But in the case of a formal noun NN-FORM, the phrase must be modified.

Phrases with a conj-pp which is exclusively used in the middle of a sentence vaux $-\mathrm{s}$, those with a conj$p p$ which is used both in the middle of a sentence and in the sentence final vaux $-s+f$, and verb phrases without any conj-pps vaux, are treated as follows:

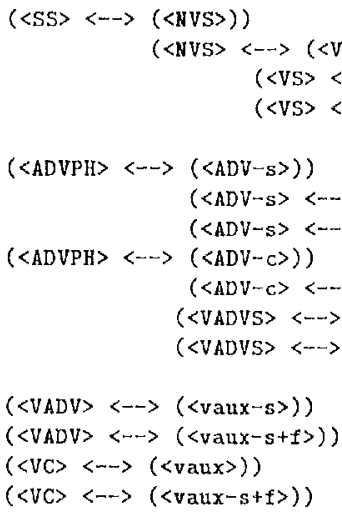

A sentence SS does not always need a noun phrase. A sentence SS can consist of only one verb phrase VC, or can be preceded by adverbial phrases ADVPH. A sentence SS can end either with a verb phrase without a conj-pps vaux or with a verb phrase with a certain kind of conj-pps vaux-s+f. An adverbial phrase ADVPH can consist of only adverbs ADV1 and can also consist of verbal phrases VaDVs. The verhal phrases

\footnotetext{
${ }^{10}$ For teminals we have a different notation. Temminals in phrase rules are phoneme strings, whose transcription is defined by the HMM-LR phonene model.

${ }^{11}$ For the sake of explanation, the rules are simplified.
} 
VADVS can contain any conj-pps, which means both vaux $\sim$ s and valux $\rightarrow \mathrm{s}+\mathrm{f}$.

Conpared with the first version, which accepts approximately $80 \%$ of the sentence candidates consisting of all the possible combinations of phrase candidates, the refined version only accepts approximately $30 \%$. Table 3 shows the size and the perplexity of the phrase rules and phrase-based sentence rules.

Table 3: Size and Perplexity of Syntactic Rules

\begin{tabular}{|c|r|r|}
\hline & Phrase lRules & Sentence Rules \\
\hline No. of liules & 1,973 & 471 \\
No. of Terminals & 744 & 133 \\
Perplexity & $3.57 /$ Phoneme & $99.7 /$ Phrase \\
\hline
\end{tabular}

\section{Validity of Rule Refinements}

We tested the improvement in two ways: speech recognition accuracy and the acceptance rate[12]. To estimate the latter we checked how many sentence candidates were filtered out by applying phrasebased sentence rules as a post-filter. We verified the rule refinements through comparison of results gained by five different rule sets: the refined version of sentence rules which contain all three refinements (Now Grammar); the refined version without conj-pp treatment (No Sentence Final Conj-pp), without formal noun treatment (No Formal Houn Treating), and without numeral treatment (No Numeral Treating); and rules which allow all combinations of phrase candidates (No Granuar). For the first four of these rule sets we determined ranks based on the probabilities of phoneme strings predicted by syntactic rules. But in the No Grammar case we determined the rank solely based on phonene probability. We experimented with the sane 353 phrases which make u]) 137 sentences as in section 2.2. 'The phrase recognition rate for the top 5 candidates was again $95.5 \%$ by exact string malching.

\subsection{Speech Recognition Accuracy}

We conducted specch recognition experiments. Figure 1 shows the constraint effectiveness of the phrasebased sentence rules given the five conditions examined. 'Ihese five conditions are compared in the graph, based on their abilities to correctly recognize the spoken sentences anong the top-ranked 20 candidates.

While the sentence recognition rate for the top candidates remains $37.2 \%$ when probability is the only factor in determining the candidates, the recognition rate rises to $70.1 \%$ when the refined syntactic rules are applied as constraints. Differentiating conj-pps is highly effective. Without this treatment, the recognition rate remains $48.2 \%$. Formal noun and numeral treatments are not as effective. Figure 1 indicates

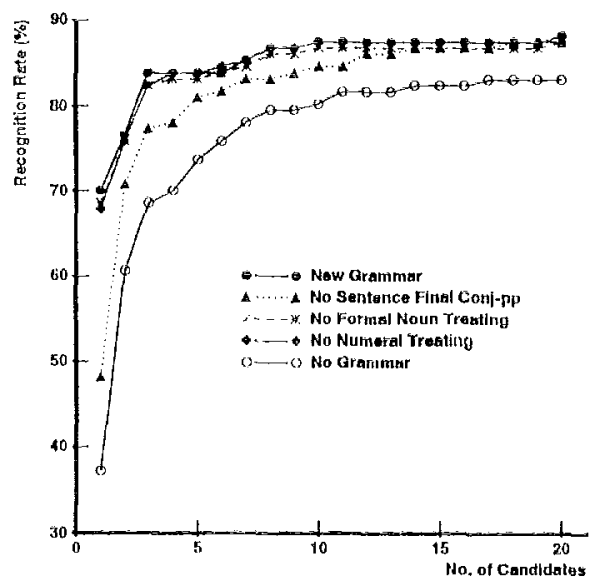

Figure 1: Comparison of Recognition Rates

that the effect according to each syntactic constraint is especially distinct up to rank 5 , and that the recognition rates saturate when we take into account sentence candidates up to rank 10 .

\subsection{Acceptance Rate}

We also verified the validity of sentence rules through the acceptance rate. We examined how many sentence candidates were filtered ont. Table 4 shows the frequencies of sentences consisting of different numbers of phrases in onr test corpus:

'liable 4: Phrase Nurnber and l'requency

\begin{tabular}{|l|r|r|r|r|r|r|r|r|}
\hline Phrase Number & 1 & 2 & 3 & 4 & 5 & 6 & 7 & 8 \\
\hline Frequency & 45 & 37 & 23 & 11 & 11 & 5 & 1 & 1 \\
\hline
\end{tabular}

Figure 2 shows the acceptance rates when applying four different syntactic rules. Whon applying rules which allow all combinations of phrase candidates, the acceptance rate remains $100 \%$.

'Ihe effect of constraints is especially clear for sentences with a small number of phrases. In senlences with one phrase, the acceptance rate for the revised version is $41 \%$, and for the version without conj-ply constraints $\% 0 \%$. In conparison with ligure 1 , treating rumerals contributes toward filtering out sentence candidates rather than raising speech recognition accuracy. Independent of the constraint strength, the more phrases there are in a sentence, the more effectively the rules work. 'ltie value for a sentence with 8 phrases is unreliable, as we have only one example. 


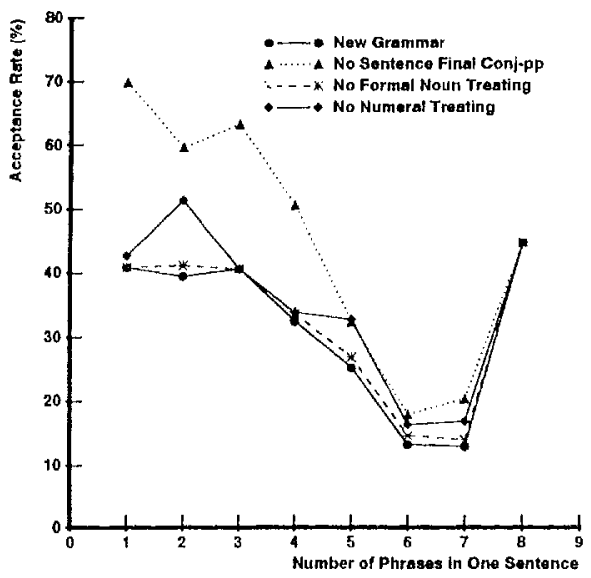

Figure 2: Acceptance Rate

\section{Conclusion}

We have described phrase-based syntactic rules which are used as constraints in the Japanese speech recognition module of our experimental speech-to-speech translation system. For constructing rules we took into account the error tendency in speech recognition. We treated precisely those syntactic categories which tend to be recognized erroneously. To increase the efficacy of each rule, the rule construction is strongly motivated by our dialogue corpus. By applying the refined phrase-based syntactic rules, the speech recognition rate for the top candidates improved from 37.2 $\%$ to $70.1 \%$ and for the top 5 candidates from 73.7 $\%$ to $83.9 \%$.

The implementation of syntactic rules based on our dialogue corpus is continuing in order to increase coverage. Currently we are studying postposition deletion in nominal phrases, which is one of the features of spoken Japanese. When adding rules and enlarging vocabulary, we cannot avoid decreasing speech recognition accuracy, but our further experiments showed that careful rule construction filtered out unacceptable sentence candidates much more effectively. Though we believe that our dialogue corpus for the. current domain provides enough expressions of spoken J apanese, we are going to apply the same method to other domains to establish the generality of the rules.

\section{Acknowledgements}

The authors wish to thank Dr.A.Kurematsu, President of ATR Interpreting Telephony Research Labs for his continued support, Mr.T.Morimoto for discussion of the various stages of this work, Mr.K.Inoue for his help in database retrieval and Dr.S.Luperfoy and Dr.L.Fais for reading an earlier draft.

\section{References}

[1] Morimoto, T., Shikano, K., lida, H., Kurematsu, A.(1990): "Integration of Speech Recognition and Language Processing in Spoken Language Translation System(SL-TRANS)," Proc. of ICSLP-90, pp.921-924.

[2] Lee, K.-F. and Hon, H.-W.(1988): "LargeVocabulary Speaker-Independent Continuous Speech Recognition Using HMM," Proc. of ICASSP-88, pp.123-126.

[3] Ney, H.(1987): "Dynamic Programming Speech Recognition Using a Context-Free Grammar," Proc. of ICASSP-87, pp.69-72.

[4] Matsunaga, S., Sagayama, S., Homma, S. and Furui, S.(1990): "A Continuous Speech Recognition System Based on a Two-Level Grammar Approach," Proc. of ICASSP-90, pp.589-592.

[5] Yamaoka, T. and Iida, H.(1990): "A Method to Predict the Next Utterance Using a Four-layered Plan Recognition Model," Proc. of ECAI-90, pp.726-731.

[6] Kuno, S. (1973): The Structure of the Japanese Language, The MIT Press, Cambridge, Massachusetts and London.

[7] Fujisaki, T. (1984): "A Stochastic A pproach to Sentence Parsing," Proc. of COLING-84, pp.16-19.

[8] Ferretti, M., Maltese, G., Scarci, S. (1990): "Measuring Information Provided by Language Model and Acoustic Model in Probabilistic Speech Recognition: Theory and Experimental Results," Speecl Communication 9, pp.531-539.

[9] Ehara, T., Ogura, K., Morimoto, T. (1990): "ATR Dialogue Database," Proc. of ICSLP-90, pp. 10931096.

[10] Kita, K., Kawabata, T, Saito, H. (1989): "HMM Continuous Speech Recogniton Using Predictive LR Parsing," Proc. of ICASSP-89, pp.703-706.

[11] Hosaka, J., Ogura, K., Kogure, K. (1990): "Word Sequence Constraints for Japanese Speech Recognition," Proc. of ECAI-90, pp. 363-365.

[12] Takezawa, T., Kita, K., Hosaka, J., Morimoto, T. (1991): "Linguistic Constraints for Continuous Speech Recognition in Goal-Directed Dialogue," Proc. of ICASSP 91, pp.801-804.

[13] Ogawa, Y., Hayashi, H., et al. (1982, 1988): Nihongo Kyoujku Jiten, Taishuukan, Tokyo, (In Japanese).

[14] Hosaka, J., Takezawa, T., Ehara, T. (1991): "Utilizing Empirical Data for Postposition Classification toward Spoken Japanese Speech Recognition," Proc. of ESCA-91, pp. 573-576. 